\title{
Author Correction: Association study in African- admixed populations across the Americas recapitulates asthma risk loci in non-African populations
}

Michelle Daya et al. ${ }^{\#}$

Correction to: Nature Communications https://doi.org/10.1038/s41467-019-08469-7, published online 20 February 2019.

The original version of this Article contained an error in the spelling of a member of the CAAPA Consortium, Hrafnhildur Bjarnadóttir which was incorrectly given as Hilda Bjarnadóttir. This has now been corrected in both the PDF and HTML versions of the Article.

Published online: 04 September 2019

\begin{abstract}
(c) (i) Open Access This article is licensed under a Creative Commons Attribution 4.0 International License, which permits use, sharing, adaptation, distribution and reproduction in any medium or format, as long as you give appropriate credit to the original author(s) and the source, provide a link to the Creative Commons license, and indicate if changes were made. The images or other third party material in this article are included in the article's Creative Commons license, unless indicated otherwise in a credit line to the material. If material is not included in the article's Creative Commons license and your intended use is not permitted by statutory regulation or exceeds the permitted use, you will need to obtain permission directly from the copyright holder. To view a copy of this license, visit http://creativecommons.org/licenses/by/4.0/.
\end{abstract}

(c) The Author(s) 2019

Michelle Daya ${ }^{1}$, Nicholas Rafaels ${ }^{1}$, Tonya M. Brunetti ${ }^{1}$, Sameer Chavan ${ }^{1}$, Albert M. Levin², Aniket Shetty ${ }^{1}$, Christopher R. Gignoux', Meher Preethi Boorgula', Genevieve Wojcik (10 ${ }^{3}$, Monica Campbell', Candelaria Vergara (10 ${ }^{4}$, Dara G. Torgerson ${ }^{5}$, Victor E. Ortega ${ }^{6}$, Ayo Doumatey ${ }^{7}$, Henry Richard Johnston ${ }^{8}$, Nathalie Acevedo9 ${ }^{9}$, Maria IIma Araujo ${ }^{10}$, Pedro C. Avila (1) ${ }^{11}$, Gillian Belbin ${ }^{12}$, Eugene Bleecker ${ }^{13}$, Carlos Bustamante ${ }^{3}$, Luis Caraballo ${ }^{9}$, Alvaro Cruz ${ }^{14}$, Georgia M. Dunston ${ }^{15}$, Celeste Eng ${ }^{5}$, Mezbah U. Faruque ${ }^{16}$, Trevor S. Ferguson (10 ${ }^{17}$, Camila Figueiredo ${ }^{18}$, Jean G. Ford ${ }^{19}$, Weiniu Gan ${ }^{20}$, Pierre-Antoine Gourraud ${ }^{21}$, Nadia N. Hansel ${ }^{4}$, Ryan D. Hernandez ${ }^{22}$, Edwin Francisco Herrera-Paz ${ }^{23}{ }^{23} 24$, Silvia Jiménez ${ }^{9}$, Eimear E. Kenny ${ }^{12}$, Jennifer Knight-Madden ${ }^{17}$, Rajesh Kumar ${ }^{25}$, Leslie A. Lange ${ }^{1}$, Ethan M. Lange ${ }^{1}$, Antoine Lizee ${ }^{21}$, Pissamai Maul ${ }^{26}$, Trevor Maul ${ }^{26}$, Alvaro Mayorga ${ }^{27}$, Deborah Meyers ${ }^{13}$, Dan L. Nicolae ${ }^{28}$, Timothy D. O'Connor ${ }^{29}$, Ricardo Riccio Oliveira ${ }^{30}$, Christopher O. Olopade ${ }^{31}$, Olufunmilayo Olopade ${ }^{28}$, Zhaohui S. Qin (1) ${ }^{32}$, Charles Rotimi (10) ${ }^{7}$, Nicolas Vince (10) ${ }^{21}$, Harold Watson ${ }^{33}$, Rainford J. Wilks ${ }^{17}$, James G. Wilson ${ }^{34}$, 


\section{Steven Salzberg (10 ${ }^{35}$, Carole Ober ${ }^{36}$, Esteban G. Burchard ${ }^{22}$, L. Keoki Williams ${ }^{37}$, Terri H. Beaty (1) ${ }^{38}$, Margaret A. Taub ${ }^{39}$, Ingo Ruczinski ${ }^{39}$, Rasika A. Mathias ${ }^{4,56}$ \& Kathleen C. Barnes ${ }^{1,56}$, CAAPA}

${ }^{1}$ Department of Medicine, University of Colorado Denver, Aurora, CO 80045, USA. ${ }^{2}$ Department of Public Health Sciences, Henry Ford Health System, Detroit, MI 48202, USA. ${ }^{3}$ Department of Genetics, Stanford University School of Medicine, Stanford, CA 94305, USA. ${ }^{4}$ Department of Medicine, Johns Hopkins University, Baltimore, MD 21224, USA. ${ }^{5}$ Department of Medicine, University of California San Francisco, San Francisco, CA 94143, USA. ${ }^{6}$ Center for Human Genomics and Personalized Medicine, Wake Forest School of Medicine, Winston-Salem 27157, USA. ${ }^{7}$ Center for Research on Genomics \& Global Health, National Institutes of Health, Bethesda, MD 20892, USA. ${ }^{8}$ Department of Human Genetics, Emory University, Atlanta, GA 30322, USA. ${ }^{9}$ Institute for Immunological Research, Universidad de Cartagena, Cartagena 130000, Colombia.

${ }^{10}$ Immunology Service, Universidade Federal da Bahia, Salvador 401110170, Brazil. ${ }^{11}$ Department of Medicine, Northwestern University, Chicago, IL 60611, USA. ${ }^{12}$ Department of Genetics and Genomics, Icahn School of Medicine at Mount Sinai, New York, NY 10029, USA. ${ }^{13}$ Department of Medicine, University of Arizona College of Medicine, Tucson, AZ 85724, USA. ${ }^{14}$ Universidade Federal da Bahia, Salvador 401110170, Brazil.

${ }^{15}$ Department of Microbiology, Howard University College of Medicine, Washington, DC 20059, USA. ${ }^{16}$ National Human Genome Center, Howard University College of Medicine, Washington, DC 20059, USA. ${ }^{17}$ Caribbean Institute for Health Research, The University of the West Indies, Kingston 00007, Jamaica. ${ }^{18}$ Departamento de Biorregulacao, Universidade Federal da Bahia, Salvador 401110170, Brazil. ${ }^{19}$ Department of Medicine, Einstein Medical Center, Philadelphia, PA 19141, USA. ${ }^{20}$ National Heart, Lung and Blood Institute, National Institutes of Health, Bethesda, MD 20892, USA. ${ }^{21}$ Université de Nantes, INSERM, Centre de Recherche en Transplantation et Immunologie, UMR, 1064ATIP-Avenir, Equipe 5, Nantes, France. ${ }^{22}$ Department of Bioengineering and Therapeutic Sciences, University of California San Francisco, San Francisco, CA 94143, USA. ${ }^{23}$ Facultad de Medicina, Universidad Católica de Honduras, San Pedro Sula 21102, Honduras. ${ }^{24}$ Universidad Tecnológica Centroamericana (UNITEC), Facultad de Ciencias Médicas, Tegucigalpa, Honduras. ${ }^{25}$ Department of Pediatrics, Northwestern University, Chicago, IL 60611, USA. ${ }^{26}$ Genetics and Epidemiology of Asthma in Barbados, The University of the West Indies, Chronic Disease Research Centre, Jemmots Lane, St. Michael BB11115, Barbados. ${ }^{27}$ Centro de Neumologia y Alergias, San Pedro Sula 21102, Honduras. ${ }^{28}$ Department of Medicine, University of Chicago, Chicago, IL 60637, USA. ${ }^{29}$ Institute for Genome Sciences, University of Maryland School of Medicine, Baltimore, MD 21201, USA. ${ }^{30}$ Laboratório de Patologia Experimental, Centro de Pesquisas Gonçalo Moniz, Salvador 40296-710, Brazil. ${ }^{31}$ Department of Medicine and Center for Global Health, University of Chicago, Chicago, IL 60637, USA. ${ }^{32}$ Department of Biostatistics and Bioinformatics, Emory University, Atlanta, GA 30322, USA. ${ }^{33}$ Faculty of Medical Sciences, The University of the West Indies, Queen Elizabeth Hospital, Bridgetown, St. Michael BB11000, Barbados. ${ }^{34}$ Department of Physiology and Biophysics, University of Mississippi Medical Center, Jackson, MS 39216, USA. ${ }^{35}$ Departments of Biomedical Engineering and Biostatistics, Johns Hopkins University, Baltimore, MD 21205, USA. ${ }^{36}$ Department of Human Genetics, University of Chicago, Chicago, IL 60637, USA. ${ }^{37}$ Center for Individualized and Genomic Medicine Research, Henry Ford Health System, Detroit, MI 48202, USA. ${ }^{38}$ Department of Epidemiology, Bloomberg School of Public Health, JHU, Baltimore, MD 21205, USA. ${ }^{39}$ Department of Biostatistics, Bloomberg School of Public Health, JHU, Baltimore, MD 21205, USA. ${ }^{56}$ These authors contributed equally: Rasika A. Mathias, Kathleen C. Barnes.

\section{CAAPA}

Ayola Akim Adegnika40, Ganiyu Arinola 41 , Ulysse Ateba-Ngoa 40 , Gerardo Ayestas ${ }^{23}$, Hrafnhildur Bjarnadóttir ${ }^{42}$, Adolfo Correa (10 ${ }^{43}$, Said Omar Leiva Erazo ${ }^{23}$, Marilyn G. Foreman ${ }^{44}$, Cassandra Foster ${ }^{4}$, Li Gao ${ }^{4}$, Jingjing Gao ${ }^{45}$, Leslie Grammer ${ }^{11}$, Mark Hansen ${ }^{46}$, Tina Hartert ${ }^{47}$, Yijuan Hu ${ }^{32}$, lain Königsberg ${ }^{1}$, Kwang-Youn A. Kim (1) ${ }^{48}$, Pamela Landaverde-Torres ${ }^{23}$, Javier Marrugo ${ }^{49}$, Beatriz Martinez ${ }^{49}$, Rosella Martinez ${ }^{23}$, Luis F. Mayorga ${ }^{23}$, Delmy-Aracely Mejia-Mejia ${ }^{50}$, Catherine Meza ${ }^{49}$, Solomon Musani ${ }^{43}$, Shaila Musharoff ${ }^{3}$, Oluwafemi Oluwole ${ }^{28}$, Maria Pino-Yanes (10 ${ }^{5}$, Hector Ramos ${ }^{23}$, Allan Saenz ${ }^{23}$, Maureen Samms-Vaughan ${ }^{51}$, Robert Schleimer ${ }^{11}$, Alan F. Scott ${ }^{52}$, Suyash S. Shringarpure ${ }^{3}$, Wei Song ${ }^{29}$, Zachary A. Szpiech (1) ${ }^{22}$, Raul Torres (1) ${ }^{53}$, Gloria Varela ${ }^{23}$, Olga Marina Vasquez ${ }^{54}$, Francisco M. De La Vega ${ }^{3}$, Lorraine B. Ware ${ }^{47}$ \& Maria Yazdanbakhsh (iD 55

\footnotetext{
${ }^{40}$ Centre de Recherches Médicales de Lambaréné, BP:242, Lambaréné 13901, Gabon. ${ }^{41}$ Department of Chemical Pathology, University of Ibadan, Ibadan 900001, Nigeria. ${ }^{42}$ Faculty of Medicine, University of Iceland, 101 Reykjavík, Iceland. ${ }^{43}$ Department of Medicine, University of Mississippi Medical Center, Jackson, MS 39216, USA. ${ }^{44}$ Pulmonary and Critical Care Medicine, Morehouse School of Medicine, Atlanta, GA 30310, USA.

${ }^{45}$ Data and Statistical Sciences, AbbVie, North Chicago, IL 60064, USA. ${ }^{46}$ Illumina, Inc., San Diego, CA 92122, USA. ${ }^{47}$ Department of Medicine, Vanderbilt University, Nashville, TN 37232, USA. ${ }^{48}$ Department of Preventive Medicine, Northwestern University, Chicago, IL 60611, USA.

${ }^{49}$ Instituto de Investigaciones Immunologicas, Universidad de Cartagena, Cartagena 130000, Colombia. ${ }^{50}$ Facultad de Ciencias de la Salud, Universidad Tecnológica Centroamericana (UNITEC), San Pedro Sula 21102, Honduras. ${ }^{51}$ Department of Child Health, The University of the West Indies, Kingston 00007, Jamaica. ${ }^{52}$ Department of Medicine, Johns Hopkins University, Baltimore, MD 21287, USA. ${ }^{53}$ Biomedical Sciences Graduate Program, University of California San Francisco, San Francisco, CA 94158, USA. ${ }^{54}$ Centro Medico de la Familia, San Pedro Sula 21102, Honduras. ${ }^{55}$ Department of Parasitology, Leiden University Medical Center, Leiden 02333, Netherlands
} 